\title{
Dynamic processes in music perception
}

\author{
CAROL L. KRUMHANSL and MARY A. CASTELLANO \\ Cornell University, Ithaca, New York
}

\begin{abstract}
A schema-based theory of music perception that describes the dynamic interaction between the musical event and the listener's knowledge of the underlying regularities in tonal music is proposed. Three properties of musical schema are evaluated in a recognition memory experiment: (1) The schema engages a subset of the abstract knowledge system that is determined by the predominant key of the musical sequence, (2) the schema evaluates both interval relations and the functions of the sounded elements within the established tonal framework, and (3) the schema interacts continuously with the musical event in time to process pitch information in its temporal context. Listeners are required to identify the serial position of a chord that is changed between two successive chord sequences that are otherwise identical. The experiment measures the magnitude and the temporal extent of the disruptive effect of including in the sequence an element outside the tonal framework. The results show temporally specific effects on memory for pitch relations consistent with the operation of a musical schema. Comparisons are made with schema-based theories applied in other perceptual and cognitive domains.
\end{abstract}

Recent theories of perception and memory have taken as a central assumption the existence and involvement of cognitive schemata. The term schema is generally used to indicate the kinds of knowledge structures that are referenced in a particular situation. Within cognitive psychology, schema-based theories have variously been applied to the perception of events, pictures, and scenes (e.g., Biederman, 1981; Mandler, 1979; Neisser, 1976; Schank \& Abelson, 1977) and to the understanding and remembering of stories and other kinds of connected discourse (e.g., Bartlett, 1932; Bower, Black, \& Turner, 1979; Kintsch \& van Dijk, 1978; Mandler \& Johnson, 1977; Rumelhart, 1975). Because the applications vary so widely both in terms of the domain of inquiry and the specific experimental situations employed to test the theoretical construct, few conclusions of a general nature have emerged from this work. Schema-based theories may be criticized because the term schema is itself only loosely defined, with the consequence that it is difficult to construct critical tests of the theories. According to Biederman (1981), "Testable empirical consequences or interesting theoretical insights have been rare. In fact, it may not be too risky to say that the primary usage of schema has been to provide a euphemism for our ignorance of the molar levels of semantic representation of pictures and stories" (p. 215). Nonetheless, the popularity and intuitive appeal of the term schema indicates that efforts directed at more precise definition and stronger empirical tests may be fruitful. In this article, we suggest how

This research was supported by a grant from the National Science Foundation (BNS-81-03570) to the first author. We are grateful to E. Goldmeier, S. Keele, and the reviewers for comments on an earlier draft. Reprint requests should be addressed to: Carol Krumhansl, Department of Psychology, Uris Hall, Cornell University, Ithaca, New York 14853. the schema construct may be employed in the context of music perception and memory, compare this usage to that in other domains, and present an experiment designed to test certain properties of musical schemata.

An initial distinction to be made is that between the kinds of abstract knowledge listeners have about musical structure and the more specific, situationally dependent knowledge that is activated by a particular musical event. In previous work (e.g., Bharucha \& Krumhansl, 1983; Krumhansl, 1979), we have referred to the former as the internal representation of musical structure, defined as the system of knowledge that listeners have about the regularities underlying the music within their experience. This knowledge is necessarily in an abstract form, and some of its characteristics are described more fully in the next section. We will refer to the subset of this knowledge that interacts dynamically with the sensory-perceptual information as a musical schema. That is, the musical schema is the subset of abstract musical knowledge that is engaged during listening; the schema is assumed to be modified by the musical event in ways that are specific to it. This usage of the term is consistent with Neisser's (1976) definition of a schema as "that portion of the entire perceptual cycle which is internal to the perceiver, modifiable by experience, and somehow specific to what is being perceived" (p. 54). It stresses the mutual interaction over time between the stimulus event and the knowledge the individual has internalized about the regularities within the domain. Schank (1980) argued that generic knowledge structures are modified or augmented by the particular situation in context-specific ways. Applying this general definition of a schema to music perception and memory requires the further specification of the kinds of abstract knowledge listeners have about musical structure, the processes through which this knowledge is referenced, 
and the consequences for perceptual organization and memory of the interaction between the abstract knowledge system and the musical event.

\section{INTERNAL REPRESENTATION OF TONAL STRUCTURE}

Previous research (summarized in Krumhansl, 1983) indicates that listeners who are familiar with Western tonal music have internalized a great deal about the structural regularities in music within that tradition. That work, as is the present article, is concerned only with pitch relations and not with other aspects, such as meter, rhythm, or larger musical forms, although these are assumed to interact with pitch organization (see, e.g., Deutsch, 1980). This work is also limited to pitch structures found in Western tonal music, a choice that is based on the availability of listeners familiar with music written in this style and the large body of music theory dealing with its structure. Bharucha and Krumhansl (1983) discuss organizational principles that may apply cross-culturally, and effects of training and development are described by Bartlett and Dowling (1980), Dowling (1982), Krumhansl and Keil (1982), Krumhansl and Shepard (1979), and elsewhere.

Traditional Western music is written so that one particular pitch, called the tonic, is emphasized; that pitch gives the name to the musical key. The tonic may function psychologically as a kind of cognitive reference point (Rosch, 1975) for the set of musical pitches as a whole (Krumhansl, 1979). As such, the tonic is the most central and structurally significant element. Music theorists describe a hierarchy of structural significance or stability, with the tonic tone occupying the highest position in the hierarchy. The position in the hierarchy of each other tone depends on the interval it forms with the tonic. This hierarchy, which has been quantitatively measured by Krumhansl and Kessler (1982) and Krumhansl and Shepard (1979), depends on a number of factors: diatonic scale membership, membership in the tonic triad chord (the chord consisting of the tonic, third, and fifth scale degrees), and mode (whether major or minor). Each key is associated with a unique hierarchy that, however, is identical under transposition to the hierarchy for other keys of the same mode. Krumhansl and Kessler (1982) demonstrated that this hierarchy is generative of structure at other levels (particularly chord-key relations and interkey distances) and argued that the hierarchy of stability that applies to the set of musical pitches is psychologically primary.

A second fundamental principle of organization in traditional Western music is the use of tones sounded simultaneously in chords. The internal representation of the harmonic functions of chords has been investigated recently (Bharucha \& Krumhansl, 1983;Krumhansl, Bharucha, \& Castellano, 1982; Krumhansl, Bharucha, $\&$ Kessler, 1982). These studies indicate that the per- ceived degree of relatedness between chords depends on whether the chords function within the basic set of harmonies of a single key. These studies show, moreover, that a hierarchy of stability also applies to the set of chords within a key. Although this hierarchy obtains independently of the key context in which the chords are embedded, other effects have been found to be context dependent. Systematic differences in the perceived relations among a fixed set of chords have been observed as the context key was varied. These differences were a regular function of interkey distance, which Krumhansl and Kessler (1982) demonstrated could be derived from the quantitative hierarchy of tonal stability. That these contextually dependent effects are functions of interkey distance suggests that the internal representation of chord functions is organized according to key structure.

These studies characterize the kind of abstract knowledge listeners have about pitch organization in tonal music. This system is rich in terms of structure at each of the three levels investigated (musical tones, chords, and keys), and interlevel influences are evident. In this account, key structure takes a central role. Each key is associated with a unique hierarchy of stability that applies to single tones and chords and can be described in terms of its relationship to every other musical key. How, then, is this internalized system of abstract musical relations referenced during listening and what are the consequences of this knowledge for perception and memory?

\section{MUSICAL SCHEMATA}

The structural description just outlined suggests that the interaction between the abstract knowledge system and the specific musical event requires that the listener derive from the pitch sequence a sense of key. This process is facilitated by the fact that tonal music employs certain conventions to establish a key. For example, the tonic tone or the tonic triad chord is typically sounded near the beginning of a musical passage, is relatively frequently sounded, and appears in rhythmically stressed positions and at the ends of major phrase boundaries. Other elements are emphasized to the extent that they are stable within the hierarchy of the key, and conventional chord sequences (cadences) are also frequently employed. These regularities suggest that listeners have developed various heuristics for achieving the sense of key. Krumhansl and Kessler (1982) investigated how the sense of key develops and changes over time during chord sequences. The results of that study indicated that a chord in isolation (the first chord of the sequence) evoked most strongly the key in which it functioned as the tonic chord; this may be considered as the default key interpretation. As subsequent chords were sounded, the sense of key was found to gradually develop and change in a way that indicated the integra- 
tion of harmonic information over multiple chords. For harmonic sequences, then, the process through which a key sense is achieved appears to be one in which the sounded chords are matched against their possible roles in the various keys. Similar processes may operate for melodic sequences, but these have not been investigated.

Once the key sense has been achieved, the appropriate subset of the abstract knowledge system may be engaged. This knowledge specifies the relations among the musical pitches, the chords, and their melodic and harmonic functions within the predominant key and possibly other, closely related keys. This, then, is the initial activation of what we have called the musical schema, the dynamic interaction between the abstract knowledge system and the particular musical event. Three properties of musical schemata are proposed here. First, only a subset of the abstract internal representation of tonal pitch structure is assumed to be engaged at any time, and this subset depends on the musical event, in particular its musical key. Second, the schema interprets the sounded elements in terms of the intervals they form and their functions within the instantiated tonal system. Third, the schema operates over time so that the interpretation of the elements in terms of interval relations and their functions within the tonal framework depends on their temporal relations to one another. Before describing the present experiment, which was designed to test each of these characteristics of musical schemata, some justification for them will be provided.

The first property proposed is that only a subset of the abstract system of tonal relations is activated and this subset is determined by the predominant key. This property is suggested by a number of previous empirical results. Bartlett and Dowling (1980) and Cuddy, Cohen, and Miller (1979) found that listeners can recognize melodies transposed (shifted in range but maintaining the relative intervals) to closely related keys more accurately than melodies transposed to distantly related keys. Dowling (1978) demonstrated, in addition, frequent confusions between a transposed melody and a tonal answer (which shifts the melody to a new range but selects the tones of the shifted melody from the key of the original sequence). Both these results suggest that the first melodic sequence engages a schema that is specific to the key of that sequence; it includes the tonal relations among pitches in that key and, to some extent, those in other closely related keys. In a similar vein, Krumhansl and Kessler (1982) found that, for chord sequences, modulations (shifts) to closely related keys were assimilated more rapidly than were modulations to more distant keys. In addition, once a key became established, it inhibited the harmonic-tonal interpretation of chords in distantly related keys below the level predicted by the individual chords in isolation. These findings indicate that the sounded elements interact with a subset of the abstract knowledge of pitch structure that is determined by the key interpretation assigned by the listener to the particular musical sequence.

The second characteristic of the musical schema proposed is that it interprets the musical elements in terms of their interval relations to one another and their melodic and harmonic functions within the predominant key. That relational information in the pitch domain is particularly central to musical schemata is suggested by a number of considerations. First, pitches per se are not identified by most listeners except those with absolute pitch. Instead, musical elements are encoded and remembered in terms of the intervals they form with other pitches in the sequence. This allows us to recognize a melody when it is transposed to a different key. Moreover, this property is consistent with music-theoretic descriptions of tonal structure in which every pitch has a well-defined function that depends on the interval it forms with the tonic tone whose placement is arbitrary. The schema is assumed not only to evaluate intervals formed by the sounded elements, but in addition and perhaps more importantly, to assign significance to the elements according to their functions within the predominant key. A variety of empirical results support this proposal. First, both melodic and harmonic sequences that conform to tonal structure are better remembered than those that do not (Bharucha \& Krumhans1, 1983; Dewar, Cuddy, \& Mewhort, 1977; Francès, 1972). Second, elements in the predominant key of tonal sequences are recognized accurately but are frequently confused with other elements within the key (Bharucha \& Krumhansl, 1983; Cuddy, Cohen, \& Miller, 1979; Dowling, 1978; Krumhansl, Bharucha, \& Castellano, 1982). Finally, a number of studies show that elements outside the prevailing key are difficult to remember. These elements, which may be described as unstable, show a marked tendency to be confused with elements that fit within the tonal framework (Bharucha \& Krumhansl, 1983; Krumhansl, 1979; Krumhansl, Bharucha, \& Castellano, 1982; Dowling \& Bartlett, Note 1). These results indicate that the musical schema evaluates both context-independent properties (such as intervals) and context-specific properties (such as the functions of the elements within the instantiated key region).

The third and final property of musical schemata proposed here is the temporal character of schemabased processing. Because music is inherently extended in time, and a great deal of its structure depends on temporal factors, little justification for this assumption seems necessary. The implications of this assumption for schema-based processing of pitch structure may need some explication, however. The primary implication is that the pitch relations are evaluated continuously as the pitches are sounded, so that relational features are processed in a time-dependent fashion. Thus, the elements are evaluated primarily in terms of their relations 
to temporally adjacent elements (see also Cuddy \& Cohen, 1976, and Deutsch, 1969). It is possible, however, that higher order structural features, such as those identified by Deutsch and Feroe (1981) and Lerdahl and Jackendoff (1977), may modify this process in certain cases by emphasizing relations formed by particular temporally separated elements and deemphasizing relations between particular temporally adjacent elements. In the absence of these higher order influences, however, the musical schema is assumed to process the pitch information in its local temporal context. That temporal order and pitch relationships interact has been demonstrated for tones (Krumhansl, 1979) and chords (Bharucha \& Krumhansl, 1983; Krumhansl, Bharucha, \& Castellano, 1982; Krumhansl, Bharucha, \& Kessler, 1982). In those studies, differences in relatedness judgments of two tones or chords were found as a function of the temporal order of the sounded elements, with the preferred order depending on the relative stability of the elements within the context key. Two other studies have shown interactions between temporal factors and pitch structure that depend on higher order structural features. Deutsch (1980) demonstrated poorer pitch memory when rhythmic and melodic phrase structure were set in opposition. More recently, Tan, Aiello, and Bever (1981) found that two-tone probes were more difficult to recognize when they straddled phrase boundaries than when they appeared in the same phrase. Little is known, however, about the dynamic character of what we have called the musical schema as it functions continuously over time.

To summarize, we propose that schemata for musical events can be described as having three properties. First, the musical event engages a subset of the listener's abstract knowledge of tonal relations that is determined by the predominant key of the musical sequence. Second, the relations among elements are evaluated by the schema for both context-independent (intervallic) and context-dependent (tonal function) features. Third, the schema operates over time to evaluate pitch relations and tonal functions in a time-dependent manner. Each of these three properties, which were investigated in the present experiment, finds some supporting evidence from previous empirical work in music perception and memory, and parallels discussed later can be found in schema-based theories applied to other domains.

\section{EXPERIMENTAL DESIGN}

The present study tested the proposed properties of musical schemata by using a short-term recognition task in which the listener heard on each trial two eightchord sequences in succession. Unlike earlier experiments on recognition memory for musical sequences, the task was not to indicate whether the two sequences were identical or different. Instead, the two sequences always differed in terms of one chord, and the listener was required to indicate which serial position contained the changed chord. This task was employed to investigate more specifically the type and temporal position of memory errors when they occur. Chord sequences were used because they permit less ambiguity of key than do melodic sequences.

The sequences were of two main types. On some trials, called All Diatonic, all the sounded chords were drawn from the basic set of harmonies of the key (the set of triads built from the tones of the diatonic scale that are therefore called diatonic). These sequences were constructed so that they would quite unambiguously instantiate a particular major key. Each sequence began with the tonic (I) chord, where the Roman numeral indicates the position of the root of the chord in the scale of the intended key. The sequence ended with a cadence in that key (either IV-I or V-I), and the chords were ordered so as to conform to conventional chord progressions (Piston, 1962, p. 18). On other trials, called One Nondiatonic, the sequences were similar in construction to All Diatonic sequences, except that they contained a single chord from outside the key. This chord, which is called nondiatonic because it is not built from the diatonic scale tones of the predominant key, was drawn from a key that was either moderately or maximally distant from the key of the sequence. On a trial, the listener heard two sequences that differed only in terms of one changed chord. On One Nondiatonic trials, the changed chord was never the nondiatonic chord; the change was always between one diatonic chord and another diatonic chord.

The main concern was to determine how the nondiatonic and changed chords affected memory for the remaining sequence elements. If, as supposed, the schema utilizes a subset of the internal representation of tonal structure determined by the predominant key, then memory for unchanged diatonic elements should be disrupted when a nondiatonic element is introduced. If the internal representation of harmonic relations is organized according to interkey distance, this disruptive effect may be stronger when the nondiatonic element is drawn from a key maximally distant from the key of the sequence than when it is drawn from a moderately distant key. Moreover, memory for the nondiatonic chord would be expected to be relatively poor because the activated schema of chord functions is supposed to be specific to the predominant and other closely related keys. If schema-based processes evaluate the relations between the sounded elements within the instantiated tonal framework, then a nondiatonic element would be poorly remembered owing to the difficulty of processing its relations to the remaining sequence elements. This would result in relatively frequent errors in which the unchanged nondiatonic would be incorrectly judged as changed. Finally, if the schema operates over time to encode relations beween temporally contiguous elements, then both the changed and nondiatonic elements 
would be expected primarily to disrupt memory for elements in their temporal vicinity. In other words, poorer recognition of unchanged diatonic elements would be expected to occur in positions near the chord actually changed on the trial and near the nondiatonic chord, when one is included in the sequence.

\section{Method}

Subjects. Sixteen subjects from the Cornell community were paid $\$ 10.00$ for participating in three experimental sessions, totaling $2.5 \mathrm{~h}$. The subjects had an average of 6.1 years of formal musical instruction. At the time, the subjects were playing a musical instrument an average of $5.3 \mathrm{~h} /$ week. Three of the subjects reported having taken one or more music theory courses at the college level. All subjects reported normal hearing and none reported absolute pitch.

Apparatus. The stimuli were generated by a Hewlett-Packard $1000 \mathrm{~L}$ computer programmed to construct a digital representation of the chords, which were played out through a HewlettPackard (59303A) digital-to-analog converter. An A.P. Circuit Corporation variable frequency filter (Model AP-255-5) was set to eliminate high-frequency noise introduced by the conversion process. Recordings were made on Maxell UD 35-90 tapes on a Revox A77 tape recorder. The tapes were played at $7 \mathrm{in.} / \mathrm{sec}$ $(19 \mathrm{~cm} / \mathrm{sec})$ on the same tape recorder through an Ampex AA620 loudspeaker.

Stimulus materials. Each chord consisted of 15 sine-wave components, with each of the three triad tones sounded in five octaves. The components were drawn from the set of equally spaced tones on a logarithmic scale with 12 semitones per octave based on $440 \mathrm{~Hz}$ (A). An amplitude envelope was imposed on the chord components so that the tones at the low and high ends of the five-octave range tapered off to threshold. Details of this method are described in Krumhansl, Bharucha, and Kessler (1982). The chords were heard at approximately $67 \mathrm{~dB}$ Each chord was sounded for $500 \mathrm{msec}$. Approximately $250 \mathrm{msec}$ separated chords within a sequence, and there was a $750-\mathrm{msec}$ pause between the end of the first (standard) sequence and the beginning of the second (comparison) sequence. Between trials, the subjects had $5 \mathrm{sec}$ to write their response. A 500 -msec burst of white noise signaled the beginning of each group of 10 trials.

Each trial consisted of two eight-chord sequences that were identical except for one changed chord in Serial Position 3, 4, 5, or 6. Trials were of two types: All Diatonic or One Nondiatonic. On All Diatonic trials, the chords were drawn from the key of $\mathrm{C}$ major, restricted to the set of relatively stable harmonic functions: I, II, IV, V, and VI. The first chord of the sequence was always the I chord, and the sequence ended with a final IV-I or V-I cadence. The sequences were constructed according to the rules of harmonic progression described by Piston $(1962$, p. 18). The One Nondiatonic trials were identical in construction, except that a single chord outside the basic set of harmonies of $\mathrm{C}$ major appeared in Serial Position 3, 4, 5, or 6 . This chord was drawn equally often from the set of I-VI chords of A major (which is moderately distant from $\mathrm{C}$ major) and $\mathrm{F} \#$ major (which is maximally distant from $\mathrm{C}$ major); the diminished VII chords were excluded. The nondiatonic chord was never the chord that was changed between the first and second sequences of the trial. This produced a total of 16 conditions, shown in Table 1. There were 24 trials in each condition, which were randomly ordered and divided into six blocks of 64 trials. Each block was preceded by three randomly selected practice trials.

Procedure. The listeners were instructed to judge which serial position contained the changed chord. They were told that only one chord would be changed and that it would be the chord in Serial Position 3, 4, 5, or 6. Also, they were told that some trials would contain a chord outside the key, but that this was
Table 1

Trial Types in Experiment

\begin{tabular}{lc}
\hline \multicolumn{1}{c}{ Sequence Type } & $\begin{array}{c}\text { Serial Position of } \\
\text { Changed Chord }\end{array}$ \\
\hline All Diatonic & $3,4,5$, or 6 \\
One Nondiatonic in Serial Position 3 & 4.5 . or 6 \\
One Nondiatonic in Serial Position 4 & 3,5 , or 6 \\
One Nondiatonic in Serial Position 5 & 3,4 , or 6 \\
One Nondiatonic in Serial Position 6 & 3,4 , or 5 \\
\hline
\end{tabular}

independent of their task. Two blocks of trials were presented in each experimental session, and each subject heard the blocks in different random orders.

\section{Results}

Correct responses. The average probability that a correct judgment about the serial position of the changed chord is shown in Figure 1. Performance was more accurate for All Diatonic trials than for sequences that contained a single chord that was nondiatonic in the predominant key $[F(2,30)=20.87, p<.001]$. On One Nondiatonic trials, there was a tendency for more errors to occur when the nondiatonic chord was drawn from the key of $\mathrm{F} \#$ major than from $\mathrm{A}$ major ( $\mathrm{F} \#$ major is more distant from the predominant key than is A major), although this difference only approached significance $[F(1,15)=2.69, p=.122]$. There was, in addition, a significant serial-position effect, such that more correct serial-position judgments were made when the changed chord was earlier in the sequence $[\mathrm{F}(3,45)=7.45$, $p<.001]$. The serial-position function did not interact

CORRECT RESPONSES

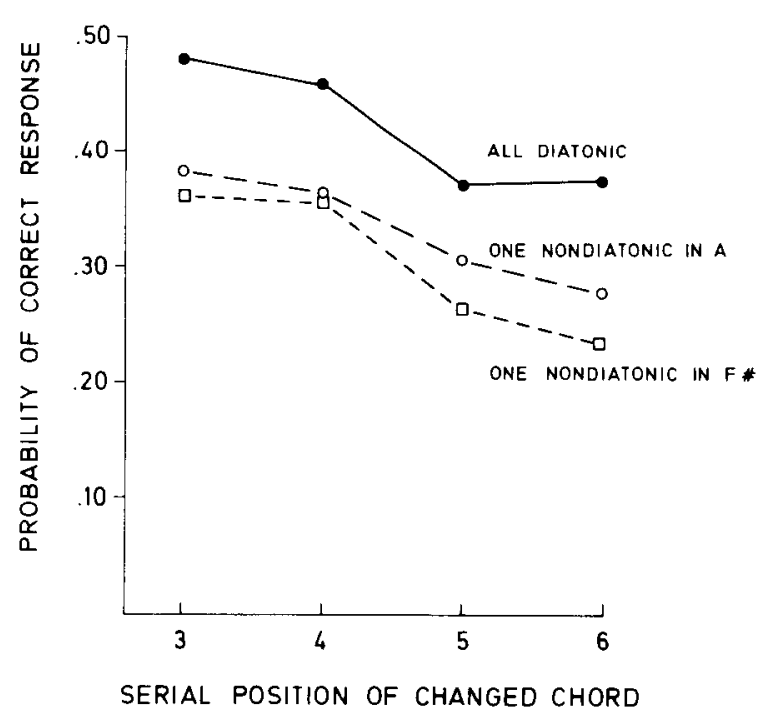

Figure 1. The percentage of correct responses as a function of the serial position of the changed chord for: All Diatonic trials, One Nondiatonic in A major trials, and One Nondiatonic in $\mathrm{F}$ \# major trials. The predominant key was always $\mathrm{C}$ major. 
with whether or not there was a nondiatonic chord in the sequence $[F(6,90)<1]$. Two additional analyses using signal-detection theory were performed. One analysis used the tables provided by Hacker and Ratcliff (1979) to convert the percent-correct scores into $d^{\prime}$ values for a four-alternative forced-choice situation The other analysis computed $\mathrm{d}^{\prime}$ values using the probability of a correct serial-position judgment as the hit rate and the probability of an incorrect serial-position judgment as the false-alarm rate for each chord position in the sequence. The statistical analyses of these $d^{\prime}$ measures produced results that were comparable to those of the analysis using the percent-correct scores.

For One Nondiatonic trials, there were no consistent differences in the probability of a correct serial-position judgment of the changed chord that depended on the number of serial positions between the changed and the nondiatonic chords. If the changed and nondiatonic chords were adjacent, the average probability of a correct response was .336 , and if the changed and nondiatonic chords were nonadjacent, the average probability of a correct response was .331 ; these values did not differ significantly $[F(1,15)<1]$. More detailed examinations of the probability of a correct response as a function of the specific combination of changed and nondiatonic chord serial positions did not reveal any regular patterns.

Error responses. Errors were those responses in which an unchanged chord was incorrectly judged as having been changed. In order to examine these errors in a way that was unconfounded by the general serial-position effect noted earlier, the probability of an error in each serial position in each condition was divided by the probability that an error occurred in that condition. This gave the conditional probability of an error in that serial position given that an error occurred in that condition. Before computing these values, the results were combined over trials with the nondiatonic chord in A and $\mathrm{F} \#$ major to stabilize the results for the One Nondiatonic trials; the results for these trials were qualitatively similar. Errors of the four types described below had different a priori probabilities of occurrence. For example, on One Nondiatonic trials, there was only one opportunity for an error to occur in a position containing a nondiatonic chord (because there was just one nondiatonic chord), but two opportunities for an error to occur in a position containing a diatonic chord. To take this into account in the error analyses, the conditional probability of an error of a given type (given that an error occurred on the trial) was divided by the number of opportunities for an error of that type. These normalized values permit comparisons of the various types of errors.

Four different error types are distinguished: (1) the total (normalized) probability of an error (in which an unchanged diatonic chord was given as the response) for All Diatonic trials, (2) the total (normalized) prob- ability of an error (in which either an unchanged diatonic or nondiatonic chord was given as the response) for One Nondiatonic trials, (3) the (normalized) probability of a diatonic error (in which an unchanged diatonic chord was given as the response) for One Nondiatonic trials, and (4) the normalized probability of a nondiatonic error (in which the unchanged nondiatonic chord was given as the response) for One Nondiatonic trials. These values are plotted on the left of Figure 2 as a function of the distance between the error serial position and the correct serial position; negative values signify that the error occurred before the chord actually changed, and positive values signify that the error occurred after the changed chord. The graph on the right shows the probability that a diatonic chord was incorrectly judged as having been changed as a function of its distance from the nondiatonic chord in the One Nondiatonic condition; negative values signify that the error occurred before the nondiatonic chord, and positive values signify that the error occurred after the nondiatonic chord.

Two effects are apparent in Figure 2 on the left. First, on One Nondiatonic trials, there was a strong tendency to incorrectly judge the nondiatonic chord as having been changed. Far more nondiatonic than diatonic errors occurred in this condition $[F(1,15)=53.51$, $p<.001]$. Second, errors of all types occurred more frequently in serial positions close to the chord that was actually changed in the trial. This effect produced an

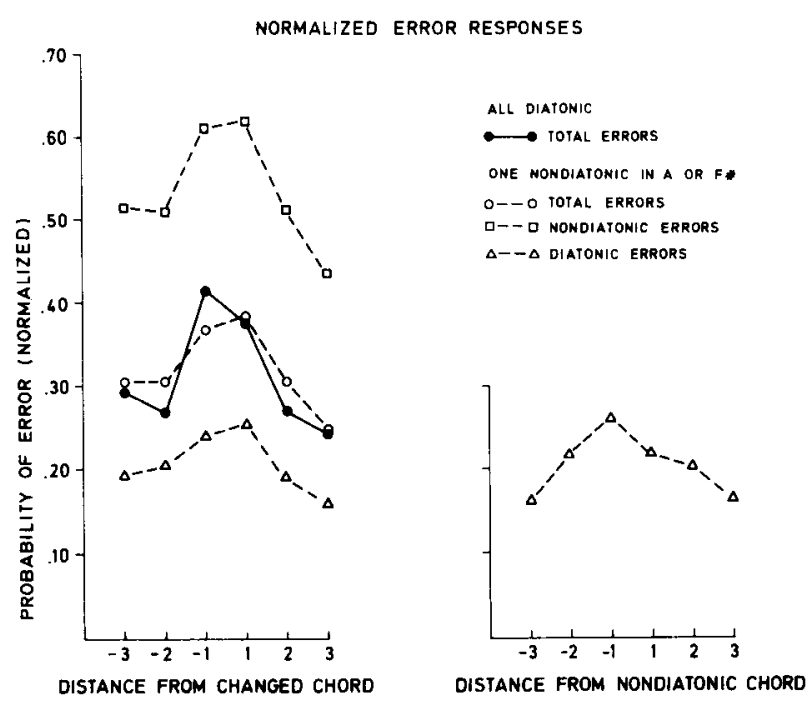

Figure 2. The probability of errors normalized by the number of errors on each trial type. The graphs on the left show errors as a function of distance from the changed chord. Total errors are shown for All Diatonic and One Nondiatonic trials, and diatonic and nondiatonic errors are shown separately for One Nondiatonic trials. The graph on the right shows diatonic errors as a function of the distance from the nondiatonic chord on One Nondiatonic trials. Negative values indicate the error occurred before the changed or nondiatonic chord; positive values indicate errors after the changed or nondiatonic chord. 
overall $\mathrm{F}(5,75)=11.92, \mathrm{p}<.001$, and was highly significant (at $p<.001$ ) for each error type when analyzed separately. The distance effect did not interact significantly with error type.

The graph on the right shows that the probability of an incorrect response was greater for serial positions close to the (unchanged) nondiatonic chord on One Nondiatonic trials $[\mathrm{F}(5,75)=4.42, \mathrm{p}<.01]$. These errors are necessarily ones in which an unchanged diatonic chord was incorrectly judged as changed. Comparing these values with the probability of a diatonic error as a function of distance from the changed chord in the plot on the left shows very similar patterns; these two functions did not interact significantly.

Finally, there was a general serial-position effect for errors of all types $[F(3,45)=5.97, p<.01]$, which did not interact with error type. More errors occurred in Serial Positions 4 and 5 than in Serial Positions 3 and 6. However, this serial-position effect can be accounted for by the fact that chords in Serial Positions 4 and 5 were, on average, closer to both the changed diatonic and the unchanged nondiatonic chord than were the chords in Serial Positions 3 and 6. Thus, the serialposition effect in the normalized errors can be attributed to the distance effects described earlier.

\section{DISCUSSION}

To summarize the results, a marked decrement in identifying the position of the changed chord occurred when the sequence contained a nondiatonic chord. There was a tendency for more errors to occur when the nondiatonic chord was drawn from a key maximally distant from the key of the sequence than when it was drawn from a key only moderately distant from the key of the sequence. In addition, an analysis of the error responses showed that much of the effect of introducing a nondiatonic chord was in the large number of errors in which the serial position of the nondiatonic chord was given as the response. That is, listeners frequently identified the nondiatonic chord as having been altered, even though it was never actually the changed chord.

An increased number of errors also occurred in the temporal vicinity of both the changed and the nondiatonic chords. Listeners relatively frequently judged chords near the changed chord as having been altered; this effect was found consistently for sequences either with or without a nondiatonic chord. When a nondiatonic chord was included in the sequence, there was also an increased number of errors in its temporal region. In both cases, the effects were found to be fairly symmetrical functions of temporal distance, and the error distributions around the changed and nondiatonic chords were similar in magnitude and shape. Finally, for all trial types, there was an increase in the number of errors when the changed chord appeared later in the sequence.
These results lend support to the proposed characterization of the musical schema, defined as the dynamic interaction between the listener's abstract knowledge of pitch relations in tonal music and the particular musical event. The musical event was presumed to engage a subset of this richly interconnected and highly structured knowledge system, and this subset is determined by the predominant key of the musical event. In the present study, introducing an element from outside the key produced marked decrements in recognition memory accuracy for chord sequences. The operation of the keyspecific musical schema, then, may be described as being disrupted by the nondiatonic chord, thus giving rise to memory errors. A slightly larger effect occurred when the nondiatonic element was drawn from a maximally distant key than from a moderately distant key. Larger effects of key distance would be expected to have been obtained had the present experiment included a wider range of key distance by including nondiatonic chords from keys closer to the predominant key. To the extent that key-distance effects are obtained, this supports the view that the sequence engages a subset of the internal representation of chord relations that is organized according to key distance, as suggested by the results of Krumhansl, Bharucha, and Castellano (1982). Other related results in the psychology of music literature were noted earlier.

The musical schema was also assumed to evaluate relations among the elements in the pitch domain, specifying not only intervallic information, but also the relations among the elements in terms of their tonal functions within the established key. In this experiment, a large number of errors occurred in the serial position containing the nondiatonic chord. These errors were far more frequent than errors in diatonic chord positions. This suggests that the musical schema operates to encode the relations among the musical elements and that this process is successful to the extent that the sounded elements fit within the established tonal framework. Again, other related effects using musical sequences have been obtained and were summarized above.

Finally, the musical schema was assumed to operate over time, dynamically interacting with the sensoryperceptual information to produce a representation in which the elements were encoded with respect to one another in a time-dependent manner. The present study found that recognition errors increased in the temporal vicinity of both the changed and the nondiatonic chords. When a changed chord appears in the sequence, it alters the relations between it and the other chords of the sequence. Since schema-based processing is presumed to evaluate pitch relations continuously in time, primarily those relations obtaining among temporally contiguous elements, unchanged chords near the changed chord would be expected to be relatively frequently judged as having been altered, and this was the result obtained. Similarly, the introduction of a nondiatonic element 
is presumed to disrupt the processing of pitch relations within its temporal region, resulting in poor relational information and the observed increase in the number of errors in positions near the nondiatonic chord. The error distribution as a function of distance from the nondiatonic chord was similar to that as a function of distance from the changed chord. This similarity may indicate that the effect of the nondiatonic element is to disrupt the schema processing of relational information in a way that is analogous to the disruption associated with the actual change in the sequence. Finally, there was a strong serial-position effect in the probability that the correct changed chord was identified. Changes earlier in the sequence were detected more frequently than were changes later in the sequence. This may be accounted for by assuming that the key-specific schema became more strongly established as the sequence progressed, causing more confusions between the changed diatonic elements when they appeared later in the sequence.

The proposed characterization of the musical schema and the present results find parallels with other schemabased approaches on both theoretical and empirical levels. It is generally held that the schema engages a system of abstract knowledge about the domain, and that this knowledge serves to process and organize information in perception and memory. An emphasis on the situationally specific nature of the schema is also found, particularly in the treatments of Neisser (1976) and Schank (1980). It should be noted that, unlike Schank's proposal, the description of the musical schema given here is not a conjunction of dissociated or loosely related elements, but a subset of the richly interconnected knowledge structure about regularities in tonal music.

The present description focuses on the central importance of relational information in the pitch domain and presumes that the schema operates to evaluate relations both in terms of context-independent (intervallic) and context-dependent (tonal-function) features. A similar emphasis on relational properties is found in the literature on scene perception, and an analogous distinction appears there between general properties that apply to all scenes, such as relations of physical support, and more content-oriented aspects of the scene schema, such as the probability that a particular object will appear in the scene (Biederman, 1981; Mandler, 1979). Effects of disrupting the normal relations have been observed in a number of studies on scene perception (e.g., Biederman, 1972, 1981; Biederman, Glass, \& Stacy, 1973; Mandler \& Johnson, 1976; Mandler \& Parker, 1976; Mandler \& Ritchey, 1977; Palmer, 1975), and effects of schema consistency on memory have been demonstrated for stories (Bartlett, 1932; Bransford \& Johnson, 1972, 1973; Mandler \& Johnson, 1977). Certain contradictory results are also found in this literature, however, and are discussed below.
The musical schema, as described here, was presumed to evaluate pitch relations among elements as they appear in the temporal sequence. Relational features in the pitch domain were assumed to be processed continuously in a time-dependent fashion. It was suggested, however, that under certain conditions the higher order structure of the sequence may modify this process. In any case, the musical schema was described as sensitive to temporal factors in its evaluation of pitch structure. Temporal ordering of the individual elements has also been proposed as one of the principal organizational properties of event and story schemas (e.g., Schank \& Abelson, 1977), and effects of violating normative temporal structure have been demonstrated in a number of studies (e.g., Bower et al., 1979; Mandler, 1978; Thorndyke, 1977). Effects of higher order structural properties have also been demonstrated in this domain (e.g., Haberlandt, Berian, \& Sandson, 1980).

Recently, Goldmeier (1982) described an extensive series of experiments concerned primarily with memory for visual forms. He found that subparts of forms that are anomalous or that deviate from structural regularity, goodness, or normativeness are poorly remembered. More important than the convergence of these results with those found in the present experiment is Goldmeier's instructive theoretical analysis. The memory trace, according to Goldmeier, "consists of coded phenomenal parts, including their functions in the whole, whereas individual elements are not necessarily coded and in general not represented in the trace" (p. 10, italics in original). Whether or not a particular element or feature will be remembered depends on its "singularity" value. If the feature fits within the global pattern, if, for example, it is suggested or implied by the remaining elements or, together with them, forms a regular, unique, or "good" pattern, then the element has a high singularity value. It will be remembered accurately, and any deviations from it will be readily detected. If, on the other hand, the singularity value of an element is low (it is "nonsingular"), then memory for that element will be poor. In particular, it will be difficult to recognize and will be confused frequently with elements that have higher singularity values. Thus, there is a narrow singularity range with high resolution and a broad range, called the nonsingular range, of low resolution. It should be emphasized that in this account, the singularity value of an element generally depends on its relations to other elements within the context, although Goldmeier notes a few cases in which singularity may be a product of innate predispositions or physiological factors. Finally, according to Goldmeier, "Once singularities are established..., they form the matrix, the schema, the framework, the filing system within which new information is assimilated" (p. 43).

Goldmeier's (1982) theoretical analysis can readily be applied to the case of memory for musical sequences considered here. The musical sequence is presumed to 
engage a musical schema that is specific to the key of the sequence. This key establishes a hierarchy of stability, or singularity, on the musical elements (the tonality-specific hierarchy described by Bharucha \& Krumhansl, 1983, Krumhansl, Bharucha, \& Kessler, 1982, Krumhansl \& Kessler, 1982, and Krumhansl \& Shepard, 1979). The nondiatonic elements, with low singularity values, have unstable memory traces. This has the consequence that nondiatonic elements are difficult to remember (in the present experiment, nondiatonic chords are frequently incorrectly judged as having been changed), and they are frequently confused with diatonic elements (Bharucha \& Krumhansl, 1983; Krumhans1, 1979; Krumhansl, Bharucha, \& Castellano, 1982; Dowling \& Bartlett, Note 1). Finally, in the cases of both music and visual form, relational information is of central importance. In fact, both in the present account and in Goldmeier's (1982) analysis, relational information predominates to the extent that the individual elements are represented primarily in terms of their relations to other elements and to the whole. On this point, Goldmeier states, "Even the parts are not necessarily represented as independent components but merely as carriers of their functions within the whole" (p. 10, italics in original).

A number of studies in the literature on text memory, however, find that anomalous or schema-inconsistent elements are better remembered than are schemaconsistent elements (Bower et al, 1979; Graesser, Gordon, \& Sawyer, 1979; Graesser, Woll, Kowalski, \& Smith, 1980; Smith \& Graesser, 1981). There are a number of possible reasons for these discrepant findings. First, in the case of stories and other kinds of text, there are a variety of different relationships that an element may have to the overall schema. It may be an event, either common or infrequent, that is simply irrelevant to the schema, or it may be an event that directly contradicts the schema structure, such as an event that interferes with a goal. These different types of schema inconsistency, once specified, may prove to have different consequences for encoding and remembering the elements. In the case of music, however, schema consistency can be defined quite unambiguously by specifying the tonal functions of the elements within the set of interrelated keys. Music has the advantage that there is a small universe of possible musical tones and chords that makes feasible the specification of the degree of consistency. A second reason for these discrepant findings may be that linguistic or semantic elements can be remembered in isolation and independently of context. That is, there are preexisting memory structures that may be referenced in a particular memory task. In contrast, memory for isolated musical elements, particularly long-term memory, is extremely poor in general; musical elements for the most part are remembered only in terms of their relationship to other elements. One final point concerns the distinction be- tween detecting and remembering an anomalous event. That memory for nondiatonic elements is poor does not imply that such elements are not noticed; indeed, quite the opposite effect is experienced. However, because the schema is unable to establish the relations between the nondiatonic element and other elements within the diatonic framework, the nondiatonic chord is only poorly remembered. In other situations in which the schema-inconsistent element can be coded and remembered independently of context, the effect may be to focus attention to and direct further processing of that element, resulting in better memory (see Biederman, 1981, for a series of related experiments on the processing of visual scenes).

To conclude, the schema-based theory of music perception and memory proposed here characterizes the listener's experience as one that is the product of integration between the ongoing pitch sequence and a system of knowledge about the structural regularities in tonal music. This process enables the listener to apprehend the structure of the composition as it is contained within the pitch and temporal relations in the music itself. It also is assumed to enable the listener to generate expectations about what is to follow. These expectations may or may not be fulfilled, or their fulfillment may be systematically delayed in time. The alteration between a sense of "tension" and a sense of "resolution" has been identified (e.g., Meyer, 1956) as one of the fundamental aspects of tonal music and as central to the aesthetic experience. Applying a schema-based theory to music perception and memory highlighted certain similarities between this and other domains, such as perceiving pictures and events and understanding and remembering stories and other kinds of connected discourse. Across domains, these theories emphasize the need to consider context-specific factors. In addition, an emphasis appears across domains on both relational properties and on temporal-order constraints. It is hoped that comparisons of this sort may serve to isolate general properties of schemata that may then be augmented by domain-specific principles as theories for the various domains develop.

\section{REFERENCE NOTE}

1. Dowling, W. J., \& Bartlett, J. C. Assimilation of brief atonal melodies to tonal prototypes: Asymmetrical effects on judgment. Paper presented at the meeting of the Psychonomic Society, Philadelphia, November 1981.

\section{REFERENCES}

Bartlett, F. C. Remembering: A study in experimental and social psychology. Cambridge: CambridgeUniversity Press, 1932.

Bartlett, J. C., \& Dowling, W. J. Recognition of transposed melodies: A key-distance effect in developmental perspective. Journal of Experimental Psychology: Human Perception and Performance, 1980, 6, 501-515.

Bharucha, J., \& Krumhanst, C. L. The representation of har- 
monic structure in music: Hierarchies of stability as a function of context. Cognition, 1983, 13, 63-102.

Biederman, I. Perceiving real world scenes. Science, 1972, 177, 77.80.

Biederman, I. On the semantics of a glance at a scene. In M. Kubovy \& J. R. Pomerantz (Eds.), Perceptual organization. Hillsdale, N. J: Erlbaum, 1981.

Biederman, I., Glass, A. L., \& Stacy, E. W., Jr. On the information extracted from a glance at a scene. Journal of Experimental Psychology, 1973, 97, 22-27.

Bower, G. H., Black, J. B., \& TuRnen, T. J. Scripts in memory for text. Cognitive Psychology, 1979, 11, 177-220.

Bransford, J. D., \& Johnson, M. K. Contextual prerequisites for understanding: Some investigations of comprehension and recall. Journal of Verbal Learning and Verbal Behavior, 1972 , 11, 717-726.

Bransford, J. D., \& Johnson, M. K. Considerations of some problems of comprehension. In W. G. Chase (Ed.), Visual information and processing. New York: Academic Press, 1973.

Cuddy, L. L., \& CohEN, A. J. Recognition of transposed melodic sequences. Quarterly Journal of Experimental Psychology, 1976, 28, 255-270.

Cuddy, L. L., Cohen, A. J., \& Miller, J. Melody recognition: The experimental application of musical rules. Canadian Journal of Psychology, 1979, 33, 148-157.

Deutsch, D. Music recognition. Psychological Review, 1969, 76, 300-307.

Deutsch, D. The processing of structured and unstructured tonal sequences. Perception \& Psychophysics, 1980, 28, 381-389.

Deutsch, D., \& Feroe, J. The internal representation of pitch sequences in tonal music. Psychological Review, 1981, 88, 503-522.

Dewar, K. M., Cuddy, L. L., \& Mewhort, D. J. K. Recognition memory for single tones with and without context. Journal of Experimental Psychology: Human Learning and Memory, $1977,3,60-67$.

Dowling, W. J. Scale and contour: Two components of a theory of memory for melodies. Psychological Review, 1978, 85, 341-354.

Dowling, W. J. Melodic information processing and its development. In D. Deutsch (Ed.), The psychology of music. New York: Academic Press, 1982.

Francès, R. La perception de la musique (2nd ed.). Paris: Librarie Philosophique J. Vrin, 1972.

Goldmeier, E. The memory trace: Its formation and its fate. Hillsdale, N.J: Erlbaum, 1982.

Graesser, A. C., Gordon, S. E., \& Sawyer, J. D. Recognition memory for typical and atypical actions in scripted activities: Tests of a script pointer + tag hypothesis. Journal of Verbal Learning and Verbal Behavior, 1979, 18, 319-332.

Graesser, A. C., Woll, S. B., Kowalski, D. J., \& Smith, D. A. Memory for typical and atypical actions in scripted activities. Journal of Experimental Psychology: Human Learning and Memory, 1980, 6, 503-515.

Haberlandt, K., Berian, C., \& Sandson, J. The episode schema in story processing. Journal of Verbal Learning and Verbal Behavior, 1980, 19, 635-650.

Hacker, M. J., \& Ratcliff, R. A revised table of d' for Malternative forced choice. Perception \& Psychophysics, 1979, 26, 168-170.

Kintsch, W., \& van DiJK, T. A. Toward a model of text comprehension and production. Psychological Review, 1978, 85, 363-394.

KRUMhansL, C. L. The psychological representation of musical pitch in a tonal context. Cognitive Psychology, 1979, 11, 346-374.

Krumhanst, C. L. Perceptual structures for tonal music. Music Perception, 1983, 1, 24-58.

Krumhansl, C. L., Bharucha, J., \& Castellano, M. A. Key distance effects on perceived harmonic structure in music. Perception \& Psychophysics, 1982, 32, 96-108.

Krumhansl, C. L., Bharucha, J. J., \& Kessler, E. J. Perceived harmonic structure of chords in three related musical keys. Journal of Experimental Psychology: Human Perception and Performance, 1982, 8, 24-36.

Krumhanse, C. L., \& KeIl, F. C. Acquisition of the hierarchy of tonal functions in music. Memory \& Cognition, 1982, 10, 243-251.

Krumhansl, C. L., \& Kessler, E. J. Tracing the dynamic changes in perceived tonal organization in a spatial representation of musical keys. Psychological Review, 1982, 89, 334-368.

Krumhansl, C. L., \& ShePard, R. N. Quantification of the hierarchy of tonal functions within a diatonic context. Journal of Experimental Psychology: Human Perception and Performance, 1979, 5, 579-594.

LERDAHL, R., \& JACKENDOFF, R. Toward a formal theory of tonal music. Journal of Music Theory, 1977, 21, 111-171.

Mandlen, J. M. A code in the node: The use of a story schema in retrieval. Discourse Processes, 1978, 1, 14-35.

Mandle R, J. M. Categorical and schematic organization in memory. In C. R. Puff (Ed.), Memory organization and structure. New York: Academic Press, 1979.

Mandler, J. M., \& Johnson, N. S. Some of the thousand words a picture is worth. Journal of Experimental Psychology: Human Learning and Memory, 1976, 2, 529-540.

Mandler, J. N., \& Johnson, N. S. Remembrance of things parsed: Story structure and recall. Cognitive Psychology, 1977, 9, 111-151.

Mandle R, J. M., \& Parker, R. E. Memory for descriptive and spatial information in complex pictures. Journal of Experimental Psychology: Human Learning and Memory, 1976, 2, 38-48.

Mandler, J. M., \& Ritchey, G. H. Long-term memory for pictures. Journal of Experimental Psychology: Human Learning and Memory, 1977, 3, 386-396.

MEYER, L. Emotion and meaning in music. Chicago: University of Chicago Press, 1956.

Ne isser, U. Cognition and reality. San Francisco: Freeman, 1976.

Palmer, S. E. The effect of contextual scenes on the identification of objects. Memory \& Cognition, 1975, 3, 519-526.

Piston, W. Harmony (3rd ed.). New York: Norton, 1962.

Rosch, E. Cognitive reference points. Cognitive Psychology, $1975,7,532-547$.

Rumelhakt, D. E. Notes on schema for stories. In D. G. Bobrow \& A. Collins (Eds.), Representation of understanding: Studies in cognitive sciences. New York: Academic Press, 1975.

Sch ANк, R. C. Language and memory. Cognitive Science, 1980, 4, 243-284.

Schank, R. C., \& Abelson, R. Scripts, plans, goals, and understanding. Hillsdale, N.J: Erlbaum, 1977.

Smith, D. A., \& Graesser, A. C. Memory for actions in scripted activities as a function of typicality, retention interval, and retrieval task. Memory \& Cognition, 1981, 9, 550-559.

Tan, N., Aiello, R., \& Bever, T. G. Harmonic structure as a determinant of melodic organization. Memory \& Cognition, 1981, 9, 533-539.

Thorndyke, P. W. Cognitive structures in comprehension and memory of narrative discourse. Cognitive Psychology, 1977, 9, 77-110. 